\title{
Autour de la relation tête-dépendant dans les langues indo-européennes anciennes. Typologie et reconstruction
}

Thèse de doctorat en Sciences du Langage, soutenue le 5 février 2016 à l'Université Lumière Lyon II, sous la direction de M. Sylvain PATRI, Professeur de Linguistique générale à l'Université Lyon II.

\section{Stefano Corno}

\section{CpenEdition}

\section{Journals}

Édition électronique

URL : http://journals.openedition.org/esp/886

DOI : $10.4000 /$ esp.886

ISSN : 2532-0319

Éditeur

Centre d'Information sur l'Éducation Bilingue et Plurilingue

Édition imprimée

Date de publication : 1 juin 2016

Pagination : 102-104

ISSN : 1127-266X

Référence électronique

Stefano Corno, « Autour de la relation tête-dépendant dans les langues indo-européennes anciennes. Typologie et reconstruction », Éducation et sociétés plurilingues [En ligne], 40 | 2016, mis en ligne le 28 octobre 2016, consulté le 30 novembre 2020. URL : http://journals.openedition.org/esp/886 ; DOI : https://doi.org/10.4000/esp.886 


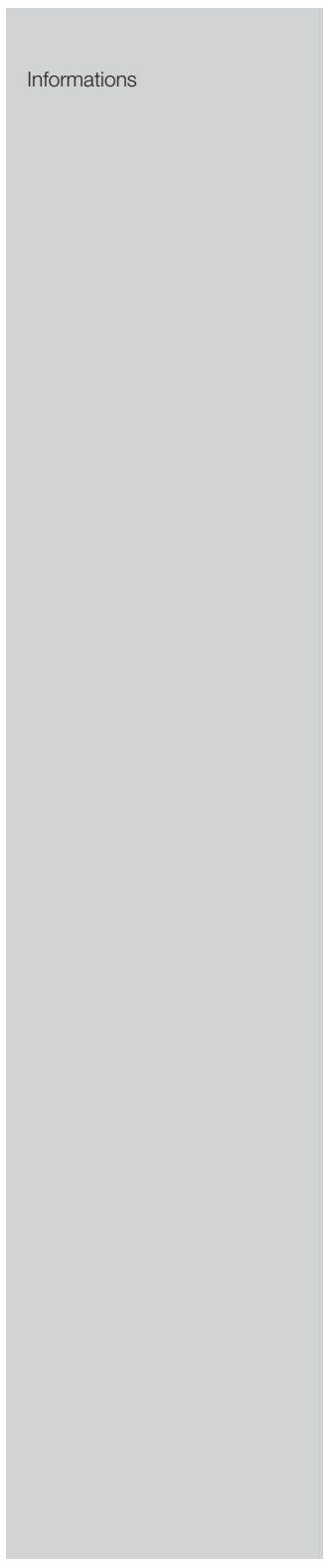

\section{Résumé de thèse}

Stefano CORNO, Autour de la relation tête-dépendant dans les langues indo-européennes anciennes. Typologie et reconstruction, Thèse de Sciences du Langage, soutenue le 5 février 2016 à l'Université Lumière Lyon II, sous la direction de M. Sylvain PATRI, Professeur de Linguistique générale à l'Université Lyon II.

Dans un constituant nominal, la tête est l'élément qui impose ses propriétés syntaxiques aux autres éléments qui composent le constituant et qui sont ses dépendants. Les dépendants adoptent les propriétés de la tête. En français, par exemple, les déterminants, démonstratifs et adjectifs inclus dans un même constituant reflètent, en principe, des propriétés imposées par le nom avec lequel ils sont en relation.

Cette thèse se propose d'étudier les relations qui se définissent entre une tête nominale et son/ses dépendant(s) à l'intérieur du constituant nominal dans les langues indo-européennes anciennes.

Du point de vue typologique, la marque de la dépendance peut être apposée sur la tête ou sur le dépendant. Dans les exemples (1) et (2), nous voyons des exemples respectivement de marquage de tête et de marquage de dépendant:
(1) a. Tadjik
${ }^{T} k_{i n h}{ }^{M} i$
b. Persan
${ }^{T}$ kûh ${ }^{M}{ }^{M}$
baland
montagne
boländ
«haute montagne»
haut

(2) Italien (indo-européen, roman)
a. ${ }^{\text {il }}$
${ }^{\mathrm{T}}$ bambino
DÉT.SGM
enfant.SGM
piccol ${ }^{-M_{O}}$
petit.SGM

«Le petit garçon»

$\begin{array}{cll}\text { b. }{ }^{M} l a & { }^{T} \text { bambina } & \text { piccol }{ }^{M} a \\ \text { DÉT.SGF } & \text { enfant.SGF } & \text { petit.SGF } \\ \text { La petite fille» } & & \end{array}$

L'exemple du tadjik et du persan moderne montre que l'élément qui porte la marque d'accord $\left({ }^{M}\right)$ est la tête ${ }^{(T)}$, tandis que celle-ci ne porte aucune marque dans l'exemple (2) car l'italien marque l'accord sur le dépendant. Il existe aussi des langues qui présentent une situation redondante, où aussi bien la tête que le dépendant sont marqués, ainsi que des langues qui ont un marquage 


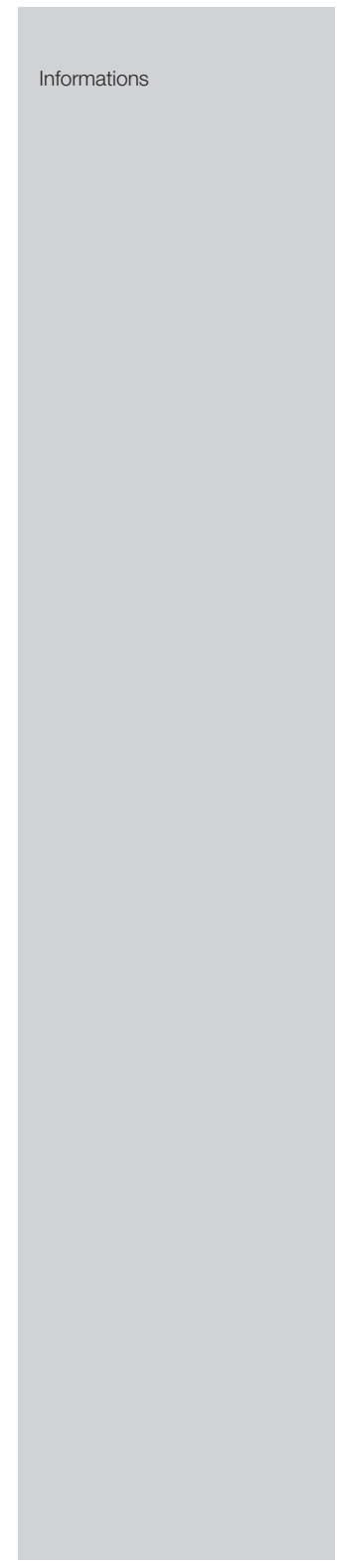

zéro, où la relation syntaxique est définie le plus souvent par l'ordre des mots.

Du point de vue typologique, le marquage du dépendant est une caractéristique plutôt homogène des langues indo-européennes anciennes, comme le souligne Nichols [1986: 89]. Pourtant, une étude approfondie de la structure de la morphologie nominale des langues indo-européennes anciennes met en évidence que l'organisation des flexions nominales diffère d'une langue à l'autre tout d'abord entre les lexèmes aptes à fonctionner comme têtes de constituants et ceux qui assument la fonction de dépendants (souvent les substantifs n'ont pas la même flexion que les adjectifs et les pronoms). De plus, même à l'intérieur de la même catégorie, tous les lexèmes ne se fléchissent pas de la même façon.

La famille linguistique indo-européenne comprend un nombre de langues trop important et trop bien attesté pour qu'on puisse rendre compte de l'intégralité des langues de cette famille dans le cadre d'une thèse de doctorat. Ainsi, un choix a dû s'opérer dans le corpus linguistique à prendre en compte. Les groupes linguistiques étudiés dans cette recherche sont les plus anciennement attestés, à savoir: indo-iranien, grec, italique et anatolien. Chacun de ces groupes comprend des langues mieux attestées et d'autres connues à un niveau plus partiel voire fragmentaire. Les langues qui ont fourni le plus de données pour chaque groupe sont les suivantes: le védique (indo-iranien), le grec homérique, le latin archaique (italique) et le hittite (anatolien).

Pour chacune de ces langues est proposée une analyse détaillée de la morphologie nominale, adjectivale et pronominale, qui permet de dégager une série de structures formelles (classes de flexion). Les différentes combinaisons possibles entre les classes de flexion des termes agissant comme tête de constituant et de celles qui fonctionnent comme dépendant permettent de dégager les différentes «classes d'accord» qui règlent la co-variation entre tête et dépendant dans la langue concernée.

Une mise en parallèle des données des quatre groupes linguistiques considérés permet d'observer une organisation différente de l'accord: on s'aperçoit, par exemple, qu'en italique le dépendant est essentiel pour définir un constituant comme masculin, puisqu'il n'existe aucune classe de flexion nominale spécifiquement masculine, tandis que cela est possible dans la flexion adjectivale et pronominale. Cela ne se produit pas dans les autres groupes.

De plus, tous ces groupes distinguent un genre neutre, destiné originellement à regrouper des noms inanimés. Or, on observe que 

tingue des autres genres, au point de permettre une double implication entre forme morphologique du nom et genre.

Mais le point le plus intéressant concerne l'anatolien, qui s'éloigne des autres groupes sous plusieurs aspects. Tout d'abord, il ne distingue que deux genres: animé et inanimé. La question se pose donc de savoir si cette organisation reflète une évolution qui verrait la fusion de masculin et de féminin dans un genre animé commun ou s'il s'agit, au contraire, d'une situation plus archaïque qui devrait être postulée comme originelle. Les analyses et les comparaisons menées tout au long de la thèse permettent de corroborer cette deuxième hypothèse, déjà largement majoritaire chez les spécialistes. Les tableaux fournis permettent également de mettre en valeur le fait qu'en indo-européen commun, le genre animé et le genre inanimé devaient disposer de désinences définies et séparées.

Mais l'anatolien présente également un aspect dans la flexion des noms inanimés qui le sépare des autres langues indo-européennes. En effet, un sujet n'est pas marqué de la même façon selon qu'il est agent d'un verbe transitif (rôle $A$ ) ou bien le participant unique d'un verbe intransitif (rôle $U$ ). En revanche, ce dernier est marqué comme l'objet (qui a le rôle de patient, rôle $P$ ).

Les noms anatoliens inanimés marquent de la même façon un sujet intransitif et un objet et réservent une marque spéciale au sujet transitif $(U, P \neq A)$. Ce type de comportement, unique dans les langues indo-européennes, est limité à la flexion des substantifs, tandis que les adjectifs et les pronoms, lorsqu'ils accompagnent un substantif inanimé dans le rôle $A$, se fléchissent au genre animé. La thèse discute ce cas apparent de discordance.

Comme nous avons déjà considéré la structure morphologique de l'anatolien comme plus ancienne que celle des autres langues de cette famille, cela nous permet de faire des inférences sur la corrélation entre le caractère d'animation et celui d'agentivité dans l'indo-européen commun. 